\title{
QUALIDADE DOS MÉIS PRODUZIDOS POR Melipona fasciculata SMITH DA REGIÃO DO CERRADO MARANHENSE
}

Carlos Alexandre Holanda, Alene Ramos Oliveira e Maria Célia Pires Costa*

Departamento de Química e Biologia, Universidade Estadual do Maranhão, Cidade Universitária Paulo VI, 65055-970 São Luís - MA, Brasil

Maria Nilce de Sousa Ribeiro

Departamento de Farmácia, Universidade Federal do Maranhão, Campus do Bacanga, 65085-580 São Luís - MA, Brasil

Janilson Lima Souza

Departamento de Química, Universidade Federal do Maranhão, Campus do Bacanga, 65085-580 São Luís - MA, Brasil

Maria José Abigail Mendes Araújo

Departamento de Microbiologia e Imunologia, Universidade Estadual Paulista, 18618-970 Botucatu - SP, Brasil

Recebido em 12/1/11; aceito em 11/5/11; publicado na web em 4/7/11

\begin{abstract}
QUALITY OF HONEY PRODUCED BY Meipona fasciculata SMITH OF CERRADO REGION FROM MARANHAO STATE, BRAZIL. The honey of Melipona fasciculata is few known in terms of composition, and therefore generally associated with the characteristics of the honey of Apis mellifera. This study contributes to the knowledge of the physico-chemical characteristics of honey of M. fasciculata of the municipalities of Barra do Corda, Jenipapo dos Vieiras, Fernando Falcão, Carolina and Riachão, in cerrado region from Maranhão. The parameters studied were: moisture, $\mathrm{pH}$, acidity, reducing sugars, apparent sucrose, hydroxymethylfurfural, diastase activity, insoluble solids, ash and color. Some of the observed patterns may conform to the established for A. mellifera, but others must be accompanied by a specific legislation.
\end{abstract}

Keywords: Melipona fasciculata; physico-chemical characteristics; cerrado region from Maranhão.

\section{INTRODUÇÃO}

A abelha sem ferrão Melipona fasciculata, encontra-se distribuída nas regiões tropicais, especialmente na América do Sul, ${ }^{1}$ e é criada há séculos pelas populações indígenas maranhenses para a produção de mel. A extração de mel dos meliponíneos é uma das possibilidades de inovação para os produtos alimentícios disponíveis no mercado, sendo capaz de ocupar a mão de obra familiar e gerar renda para pequenos proprietários rurais. ${ }^{2}$ No Estado do Maranhão, M. fasciculata, popularmente conhecida como tiúba, tem uma importante inserção no mercado local, produzindo um mel de qualidade e com amplas possibilidades para exploração em escala econômica. O mel de tiúba, por ser mais líquido, ácido e de cor suave que o mel de abelhas do gênero Apis, alcança preços muito mais elevados. ${ }^{3}$

Entretanto, são relativamente recentes os estudos que descrevem as características físico-químicas do mel de meliponíneos, sendo de fundamental importância a caracterização de méis de regiões tropicais, onde a flora é bastante diversificada, associada às taxas elevadas de umidade e temperatura. ${ }^{4} \mathrm{O}$ mel de $M$. fasciculata possui um aroma bastante atrativo devido à florada e às características biológicas das abelhas que elevam a concentração de açúcar até o máximo de $74 \%$. É muito comercializado na região da baixada maranhense, contudo ainda existem barreiras comerciais que impedem o pleno sucesso da meliponicultura no Estado, principalmente devido ao desconhecimento do perfil de qualidade do produto. ${ }^{5,6}$ Há necessidade de caracterizar estes méis para fins de comercialização, pois a legislação brasileira só regulamenta o mel produzido pelas abelhas do gênero Apis, não contempla as abelhas nativas, o que leva à necessidade de estudos para a padronização de diferentes méis produzidos por meliponíneos. ${ }^{7}$

O presente trabalho teve como objetivo contribuir para o esta-

*e-mail: celiacosta@prof.elo.com.br belecimento das características físico-químicas do mel de Melipona fasciculata produzido na região do cerrado maranhense e comparar a qualidade deste mel com os padrões estabelecidos pela legislação para os méis de Apis mellifera.

\section{PARTE EXPERIMENTAL}

\section{Coleta das amostras}

14 amostras de méis de Melipona fasciculata foram analisadas, em porções de aproximadamente $250 \mathrm{~g}$. As coletas foram feitas com seringas esterilizadas e as amostras acondicionadas em frascos de vidro estéreis à temperatura ambiente, nos municípios de Barra do Corda (4 amostras), Jenipapo dos Vieiras (3 amostras), Fernando Falcão (3 amostras), Carolina (2 amostras) e Riachão (2 amostras), pertencentes à região do cerrado maranhense com florada diversificada. Todas as amostras de méis foram obtidas em conjunto e de forma asséptica pelos autores e produtores diretamente das colmeias, nos meses de setembro e outubro de 2007., ${ }^{8,9}$

\section{Análises físico-químicas}

A pesquisa foi realizada no Laboratório de Macromoléculas e Produtos Naturais da Universidade Estadual do Maranhão, São Luís, MA. Os parâmetros determinados foram umidade, $\mathrm{pH}$, acidez, açúcares redutores, sacarose aparente, hidroximetilfurfural (HMF), atividade diastásica, sólidos insolúveis, cinzas ${ }^{10,11}$ e classificação da cor. ${ }^{12}$

A umidade foi determinada por meio do índice de refração utilizando-se um refratômetro Abbé calibrado; os valores obtidos foram convertidos em percentuais de umidade. A acidez (total) foi determinada por titulação com hidróxido de sódio $\left(0,1 \mathrm{~mol} \mathrm{~L}^{-1}\right)$ com ponto final em $\mathrm{pH} 8,3$. Os açúcares redutores foram determinados por titulação volumétrica utilizando os reativos Fehling A e B, como 
indicador, o azul de metileno. A sacarose aparente foi obtida pela diferença dos açúcares totais (redução por ácido sulfúrico) e redutores. As análises de HMF, atividade diastásica e cor foram determinadas por espectrofotometria na região do visível, enquanto sólidos insolúveis e cinzas por gravimetria.

\section{Análise estatística}

Os resultados foram analisados com auxílio do software Graph Pad Prism, versão 5.0. Os dados foram expressos com a média \pm desvio padrão (D.P.) de 3 repetições. Essas informações foram comparadas com os padrões estabelecidos pela legislação brasileira para os méis de abelhas do gênero Apis mellifera. ${ }^{13}$

\section{RESULTADOS E DISCUSSÃO}

As amostras de mel produzidas por Melipona fasciculata apresentaram um teor de umidade variando de 21,44 a 27,51\% (23,80 \pm $1,90 \%$ ) (Tabela 1). Esses valores estão de acordo com CortopassiLaurino e Gelli, ${ }^{14}$ que constataram que a umidade do mel de meliponíneos pode variar de 18 a $35 \%$. Oliveira et al. ${ }^{9}$ analisaram méis produzidos por M. fasciculata obtidos no Estado do Maranhão e observaram variações no teor de umidade dependendo da região: mínimo de $21,44 \%$ e máximo de $27,51 \%$ para os méis do cerrado maranhense; mínimo de $22,60 \%$ e máximo de $32,60 \%$ para os méis da baixada maranhense. O excesso de água encontrado nos méis de meliponíneos pode ser devido à baixa taxa de desidratação do néctar durante o processo de transformação em mel. Espécies de habitats úmidos geralmente apresentam conteúdo de água mais elevado quando comparados às de ambiente seco, o que indica a influência das condições ambientais. ${ }^{15} \mathrm{O}$ teor de umidade encontrado nos méis produzidos por diversas espécies de meliponíneos é elevado quando comparado ao valor estabelecido para o mel de abelhas do gênero Apis (máximo 20\%) ${ }^{13} ; 25-31 \%$ para M. quadrifasciata; $26-31 \%$ para
M. scutellaris; $23-32 \%$ para M. tetragonisca angustula; $27-32 \%$ para M. asilvai; 25-29\% para M. compressipes manoense; $25-35 \%$ para M. seminigra merribae; $23-32 \%$ para $M$. mandaçaia. ${ }^{7,16-20}$

As medidas de $\mathrm{pH}$ obtidas variaram de 3,1 a 4,4 (3,6 $\pm 0,4)$ (Tabela 1). Os valores de $\mathrm{pH}$ para o mel de meliponíneos não estão padronizados pela legislação nacional ou internacional, mas é estipulada uma faixa de 3,3 a 4,6 para Apis mellifera. ${ }^{17}$ Normalmente o pH dos méis é baixo, sendo que méis com origem botânica definida possuem características distintas de $\mathrm{pH}$, sendo este um fator importante, por influenciar na velocidade de formação do HMF. ${ }^{21}$ Os valores de acidez para as amostras analisadas variaram de 22,78 a 42,67 meq kg-1 (31,88 \pm 7,09 meq kg-1) (Tabela 1). Todos os valores encontrados estão em conformidade com as normas nacionais para méis de abelhas do gênero Apis. ${ }^{13}$

O teor médio de acidez das amostras encontra-se próximo ao encontrado por Souza e Bazlen ${ }^{21}$ em méis de Melipona fasciculata do Piauí (45,75 meq kg-1), enquanto Souza et al. ${ }^{18}$ encontraram média de 41,64 meq kg ${ }^{-1}$ em méis do estado da Bahia. A quantidade de íons hidrogênio que determina a acidez do mel de tiúba (baixo valor de $\mathrm{pH}$ ) é um importante parâmetro para a manutenção da estabilidade química, pois minimiza o risco de desenvolvimento de micro-organismos. ${ }^{22}$

O conteúdo de açúcares redutores obtido variou de 50,01 a $65,79 \%(60,68 \pm 4,12 \%)$ (Tabela 1$)$. Carvalho ${ }^{23}$ obteve média de $64,70 \%$ analisando amostras de méis de meliponíneos. A legislação nacional estabelece mínimo de $65 \%$ para açúcares redutores em méis de A. mellifera, entretanto o resultado médio obtido para as amostras de $M$. fasciculata se encontra abaixo do parâmetro exigido para $A$. mellifera. ${ }^{2}$ Méis de meliponíneos possuem menor teor de açúcares e normalmente a frutose é predominante, sendo um dos fatores responsáveis pela doçura do mel e sua higroscopicidade. ${ }^{9} \mathrm{O}$ teor mínimo de açúcares redutores para o mel de tiúba, assim como as outras variáveis físico-químicas, ainda não estão regulamentadas. O mel de tiúba é um produto cuja concentração de açúcares totais está entre 70-74\%, sendo inferior aos $80 \%$ normalmente detectados nos méis de abelhas

Tabela 1. Valores da média e do desvio padrão de umidade, $\mathrm{pH}$, acidez, açúcares redutores e sacarose aparente determinados nas amostras de méis de Melipona fasciculata do cerrado maranhense

\begin{tabular}{|c|c|c|c|c|c|}
\hline Amostra & Umidade (\%) & $\mathrm{pH}$ & Acidez (meq kg-1) & $\operatorname{AR}^{\ddagger}(\%)$ & $\mathrm{SA}^{\dagger}(\%)$ \\
\hline M1 BC* & 21,85 & 3,5 & 33,49 & 60,14 & 2,46 \\
\hline M2 BC* & 21,45 & 3,4 & 24,14 & 60,99 & 3,02 \\
\hline M3 BC* & 22,65 & 3,7 & 24,65 & 50,01 & 6,08 \\
\hline M4 BC* & 21,44 & 3,6 & 42,67 & 63,57 & 3,11 \\
\hline M5 JV** & 25,85 & 3,1 & 28,56 & 59,56 & 1,47 \\
\hline M6 JV** & 26,25 & 3,1 & 38,76 & 59,04 & 3,45 \\
\hline M7 JV** & 23,98 & 3,2 & 22,78 & 55,99 & 2,84 \\
\hline $\mathrm{M} 8 \mathrm{FF}^{* * *}$ & 27,51 & 3,2 & 27,88 & 59,55 & 2,95 \\
\hline M9 $\mathrm{FF}^{* * *}$ & 23,31 & 4,4 & 23,12 & 63,60 & 3,37 \\
\hline $\mathrm{M} 10 \mathrm{FF}^{* * *}$ & 22,65 & 4,2 & 34,17 & 65,79 & 0,89 \\
\hline $\mathrm{M} 11 \mathrm{C} * * * *$ & 22,71 & 4,4 & 40,03 & 65,78 & 1,19 \\
\hline $\mathrm{M} 12 \mathrm{C}^{* * * *}$ & 25,05 & 3,6 & 41,48 & 60,01 & 1,74 \\
\hline $\mathrm{M} 13 \mathrm{R} * * * * *$ & 23,24 & 3,6 & 36,00 & 63,57 & 1,65 \\
\hline $\mathrm{M} 14 \mathrm{R} * * * * *$ & 25,24 & 3,2 & 28,67 & 61,99 & 2,95 \\
\hline V. Padrão & máx. 20,00 & $3,3-4,6$ & máx. 50,00 & mín. 65,00 & máx. 6,00 \\
\hline Média & 23,80 & 3,6 & 31,88 & 60,68 & 2,65 \\
\hline Desvio & 1,90 & 0,4 & 7,09 & 4,12 & 1,30 \\
\hline
\end{tabular}

*BC - Barra do Corda; **JV - Jenipapo dos Vieiras; ***FF - Fernando Falcão; ****C - Carolina; *****R - Riachão. ${ }^{*}$ AR - Açúcares redutores; ${ }^{*}$ SA - Sacarose aparente. 
do gênero Apis. ${ }^{2} \mathrm{O}$ sabor dos méis das abelhas nativas é influenciado pelo baixo teor de açúcares e $\mathrm{pH}$ ácido, o que denota uma notável preferência do consumidor por este tipo de mel. ${ }^{23} \mathrm{~A}$ porcentagem de sacarose aparente das amostras analisadas variou de 0,89 a $6,08 \%$ $(2,65 \pm 1,30 \%)$ (Tabela 1$)$. A legislação para méis de A. mellifera estabelece um valor máximo de $6 \% .{ }^{4}$ Há uma grande variação na distribuição da sacarose nas amostras de mel nos diversos trabalhos encontrados na literatura, ${ }^{2}$ dentre estes podemos citar o trabalho de Souza et al. ${ }^{18}$ que obtiveram média de $4,70 \%$ em méis de $M$. asilvai, Alves et al. ${ }^{7}$ 2,91\% em méis de $M$. mandaçaia e Almeida-Anacleto et al. ${ }^{17} 0,95 \%$ em méis de $M$. tetragonisca angustula.

Os teores de HMF variaram de 5,44 a 70,79 $\mathrm{mg} \mathrm{kg}^{-1}(27,38 \pm$ $19,32 \mathrm{mg} \mathrm{kg}^{-1}$ ) (Tabela 2). 92,86\% das amostras estão abaixo do valor máximo estabelecido pela legislação nacional $\left(60 \mathrm{mg} \mathrm{kg}^{-1}\right)$ e internacional ( $80 \mathrm{mg} \mathrm{kg}^{-1}$, países tropicais) para méis de abelhas do gênero Apis. ${ }^{24} \mathrm{O}$ HMF encontrado em amostras de méis de outras espécies de Melipona também apresentou valores baixos, como em M. asilvai $\left(2,44 \mathrm{mg} \mathrm{kg}^{-1}\right),{ }^{18}$ M. mandaçaia $\left(5,79 \mathrm{mg} \mathrm{kg}^{-1}\right)^{7} \mathrm{e}$ M. tetragonisca angustula $\left(9,39 \mathrm{mg} \mathrm{kg}^{-1}\right) .{ }^{17}$ Sabe-se que a legislação brasileira foi estabelecida para o mel de abelha Apis, mas neste item, o mel de abelha nativa também pode se enquadrar, tendo em vista que o $\mathrm{HMF}$ apresentou valores abaixo de $60 \mathrm{mg} \mathrm{kg}^{-1}$ de mel. Esta substância é formada pela reação de certos açúcares com ácidos, sendo a frutose considerada a principal formadora do composto, devido à ação de ácidos e do calor. ${ }^{25}$

Os índices de diastase encontrados nas amostras variaram de 0,60 a 2,93 Gothe $(1,43 \pm 0,77$ Gothe) (Tabela 2$)$. Todas as amostras analisadas apresentaram quantidades enzimáticas inferiores ao valor estabelecido pela legislação (8 Gothe), mesmo utilizando a correlação com HMF ( 3 Gothe para valores de HMF menores que $15 \mathrm{mg} \mathrm{kg}^{-1}$ ). ${ }^{26,27}$ Os valores encontrados neste trabalho corroboram os encontrados por Guerrini et al. ${ }^{28}$ que obtiveram um valor médio de 1,60 Gothe para méis produzidos por meliponíneos da região amazônica do Equador, e Carvalho et al.,${ }^{16}$ que encontraram uma variação de 1,40 a 2,14 e 2,16 a 3,01 Gothe para méis produzidos por $M$. scutellaris e $M$. quadrifas- ciata, respectivamente. Souza et al. ${ }^{29}$ analisaram a atividade diastásica em várias espécies de abelhas sem ferrão, obtendo uma variação de 0,9 a 23,0 Gothe. A ocorrência de grandes diferenças quantitativas dessa enzima em méis de diferentes origens florais sugere possíveis efeitos de substâncias provenientes da flora (néctar) e/ou naturais do mel. A grande variação enzimática observada por White Júnior em méis recém-colhidos, levou-o a questionar o uso da atividade diastásica para qualificar a procedência do mel. ${ }^{30}$

Os teores de sólidos insolúveis variaram de 0,01 a $0,11 \%(0,02$ $\pm 0,02 \%)$. As amostras analisadas encontram-se dentro dos padrões estabelecidos pela legislação, que estabelece um teor máximo de sólidos insolúveis em água de $0,1 \%$, tanto para o mel de flores como para o de melato. ${ }^{14}$ Os valores aproximam-se dos observados por Evangelista-Rodrigues et al., ${ }^{31}$ que obtiveram média de 0,01\% para o mel de M. scutellaris obtido na região do Brejo Paraibano. Baixos valores de sólidos insolúveis evidenciam o caráter higiênico das abelhas na produção do mel; os resultados qualificam as abelhas tiúbas como higiênicas quanto à produção do seu mel. ${ }^{32}$ Os resultados de cinzas estão dentro do estabelecido para os méis de abelhas do gênero Apis (0,6\%), apresentando uma variação de 0,02 a 0,57\% (0,21 \pm $0,15 \%$ ) (Tabela 2). ${ }^{32} \mathrm{~A}$ variação nos teores de cinzas pode ser associada à diversidade foral do cerrado maranhense, que é diretamente associada aos minerais presentes no solo. ${ }^{4,33}$ Elevados percentuais de cinzas caracterizam o mel como rico em minerais. ${ }^{32}$

A análise do mel de $M$. fasciculata demonstrou uma grande variedade de cores, predominando o extra âmbar claro e âmbar claro na maior parte das amostras. As colorações obtidas assemelham-se às encontradas por Almeida-Anacleto et al. ${ }^{17}$ para os méis de $M$. tetragonisca angustula (predominância do extra âmbar claro) e por Alves et al. ${ }^{7}$ para os méis de $M$. mandaçaia (predomínio do âmbar claro). As amostras analisadas estão dentro dos padrões exigidos pela legislação brasileira, que classifica o mel do incolor (branco d'água) ao âmbar escuro. ${ }^{4}$ No mercado mundial, o mel é avaliado por sua cor, méis mais claros alcançam um preço mais alto que os de cor escura. A variedade de cor nos méis está interligada à sua origem floral, no

Tabela 2. Valores da média e do desvio padrão de HMF, atividade diastásica, sólidos insolúveis, cinzas e cor determinados nas amostras de méis de Melipona fasciculata do cerrado maranhense

\begin{tabular}{|c|c|c|c|c|c|}
\hline Amostra & $\mathrm{HMF}\left(\mathrm{mg} \mathrm{kg}^{-1}\right)$ & Diastase (Gothe) & $\mathrm{SI}^{\dagger}(\%)$ & Cinzas $(\%)$ & Cor \\
\hline M1 BC* & 43,96 & 1,02 & 0,01 & 0,17 & E. A. C \\
\hline M2 BC* & 48,64 & 0,99 & 0,01 & 0,15 & Âmbar claro \\
\hline M3 BC* & 24,91 & 1,48 & 0,02 & 0,18 & Branco \\
\hline M4 BC* & 5,44 & 2,93 & 0,02 & 0,16 & Âmbar \\
\hline M5 JV** & 13,17 & 0,86 & 0,01 & 0,02 & Branco \\
\hline M6 JV** & 17,77 & 1,12 & 0,01 & 0,14 & E. A. $C^{\ddagger}$ \\
\hline M7 JV** & 16,80 & 0,84 & 0,02 & 0,19 & E. A. C \\
\hline M8 $\mathrm{FF}^{* * * *}$ & 9,06 & 0,83 & 0,03 & 0,16 & E. A. C \\
\hline M9 $\mathrm{FF}^{* * *}$ & 35,13 & 1,52 & 0,11 & 0,42 & Âmbar claro \\
\hline M10 FF*** & 44,41 & 0,60 & 0,03 & 0,39 & Âmbar claro \\
\hline M11 C***** & 70,79 & 1,02 & 0,01 & 0,57 & Âmbar \\
\hline M12 C $* * * * *$ & 33,69 & 1,51 & 0,02 & 0,13 & Âmbar claro \\
\hline M13 R***** & 10,65 & 2,71 & 0,01 & 0,10 & Âmbar \\
\hline M14 R****** & 8,92 & 2,65 & 0,02 & 0,08 & Branco \\
\hline V. Padrão & máx. 60,00 & mín. 8,00 & máx. 0,10 & máx. 0,60 & B. A. $E^{ \pm}$ \\
\hline Média & 27,38 & 1,43 & 0,02 & 0,21 & \\
\hline Desvio & 19,32 & 0,77 & 0,02 & 0,15 & \\
\hline
\end{tabular}

*BC - Barra do Corda; **JV - Jenipapo dos Vieiras; ***FF - Fernando Falcão; ****C - Carolina; *****R - Riachão. ${ }^{\dagger}$ SI - Sólidos insolúveis; ${ }^{*}$ E.A.C - Extra âmbar claro; ${ }^{ \pm}$B.A.E - Branco d'água a âmbar escuro. 
entanto, os compostos responsáveis pela coloração ainda não têm sua estrutura conhecida.?

\section{CONCLUSÃO}

As análises físico-químicas das amostras de mel de Melipona fasciculata, coletadas no cerrado maranhense, atendem à maioria dos pré-requisitos estabelecidos pela legislação brasileira para méis de Apis (acidez, sacarose aparente, HMF, sólidos insolúveis, cinzas e cor).

A umidade, o teor de açúcar e a atividade diastásica necessitam de padrões que regulamentem os valores encontrados para méis de meliponíneos. Principalmente a umidade, que aumenta a probabilidade de deteriorização por micro-organismos. No entanto, a elevada acidez deste mel pode minimizar este fator negativo.

\section{AGRADECIMENTOS}

À Fundação de Amparo à Pesquisa do Estado do Maranhão (FAPEMA) pela concessão da bolsa de iniciação científica para C. A. Holanda, no período 2006/2007; ao Banco do Nordeste, pelo auxílio concedido ao projeto: "Controle de qualidade químico e biológico como fator para certificação do mel e da geoprópolis de Melipona fasciculata Smith (tiúba) da região do cerrado maranhense", e aos meliponicultores: W. C. Moreira, I. Vieira, O. Cavalcante, J. Dias, F. Pires, L. Guedes, J. de R. de Sá, J. de Barros, J. Barros, B. Álvaro, D. Carreiro, C. de Oliveira e A. de Jesus, pela doação das amostras de mel.

\section{REFERÊNCIAS}

1. Silveira, F. A.; Melo, G. A. R.; Almeida, E. A. B; Abelhas Brasileiras: Sistemática e Identificação, Composição e Arte: Belo Horizonte, 2002.

2. Nogueira Neto, P. A.; Vida e Criação de Abelhas Indígenas sem Ferrão, Nogueirapis: São Paulo, 1997.

3. Kerr, W. E.; Biologia e Manejo da Tiúba, a Abelha do Maranhão, EDUFMA: São Luís, 1996.

4. Mendonça, K.; Marchini, L. C.; Souza, B. de A.; Almeida-Anacleto, D. de; Moreti, A. C. de C. C.; Cienc. Rural 2008, 38, 1748.

5. Faria, J. A. F.; Inf. Agropec. 1983, 9, 61.

6. Kerr, W. E.; Resumos do Congresso Brasileiro de Apicultura, Teresina, Brasil, 1996.

7. Alves, R. M. O.; Carvalho, C. A. L.; Souza, B. A.; Sodré, G. S.; Marchini, L. C.; Ciênc. Tecnol. Aliment. 2005, 25, 644.

8. Oliveira, E. G.; Costa, M. C. P.; Nascimento, A. R.; Monteiro Neto, V.; Higiene Alimentar 2005, 133, 92.

9. Oliveira, E. G.; Silveira, L. M. da S.; Nascimento, A. R.; Monteiro Neto, V.; Nahuz, M. do S. R.; Meneses, S. L. de; Vasconcelos, A. F. F. de; Borges, A. C. S.; Bogéa, A. L. G.; Azevedo, C. C. de; Ferreira, C. F. C.; Lima, J. C.; Costa, M. C. P.; Higiene Alimentar 2006, 20, 74.
10. Codex Alimentarius Commission; Codex Alimentarius, 1990

11. Association of Official Analytical Chemists; Official methods of analysis, Arlington, 1998.

12. Della Modesta, R. C.; Manual de análise sensorial de alimentos e bebidas: prática, EMBRAPA/CTAA: Rio de Janeiro, 1994.

13. http://www.engetecno.com.br/port/legislacao/mel_mel_rtfiq.htm, acessada em Junho 2011.

14. Cortopassi-Laurino, M.; Gelli, D. S.; Apidologie 1991, 22, 61.

15. Cortopassi-Laurino, M.; Montenegro, A.; Resumos do XIII Congresso Brasileiro de Apicultura, Florianópolis, Brasil, 2000.

16. Carvalho, C. A. L.; Sodré, G. S.; Fonseca, A. A. O.; Alves, R. M. O.; Souza, B. de A.; Clarton, L.; An. Acad. Bras. Cienc. 2009, 81, 143.

17. Almeida-Anacleto, D. de; Souza, B. de A.; Marchini, L. C.; Moreti, A. C. de C. C.; Ciênc. Tecnol. Aliment. 2009, 29, 535.

18. Souza, B. de A.; Carvalho, C. A. L.; Sodré, G. S.; Marchini, L. C.; Cienc. Rural. 2004, 34, 1623.

19. Almeida-Muradian, L. B.; Matsuda, A. H.; Bastos, D. H. M.; Quim. Nova 2007, 30, 707.

20. Souza, R. C.; Yuyama, L. K. O.; Aguiar, J. P. L.; Oliveira, F. P. M.; Acta Amaz. 2004, 34, 333.

21. Souza, D. C.; Bazlen, K.; Resumos do XII Congresso Brasileiro de Apicultura, Salvador, Brasil, 1998.

22. Evangelista, R. A.; Silva, E. M. S.; Bezerra, E. M. F.; Rodrigues, M. L.; Cienc. Rural. 2005, 35, 1166.

23. Carvalho, L. S.; Monografia de Graduação, Universidade Estadual do Maranhão, São Luís, Brasil, 2001.

24. Lemos, G. da S.; dos Santos, J. S.; dos Santos, M. L. P.; Quim. Nova 2010, 33, 1682

25. White Júnior, J. W.; American Bee J. 1992, 132, 792.

26. Özcan, M.; Arslan, D.; Ceylan, D. A.; Food Chem. 2006, 99, 24

27. Persano-Oddo, L.; Heard, T. A.; Rodríguez-Malaver, A.; Pérez, R. A.; Fernández-Muiño, M.; Sancho, M. T.; Sesta, G.; Lusco, L.; Vit, P.; J. Med. Food 2008, 11, 789.

28. Guerrini, A.; Bruni, R.; Maietti, S.; Poli, F.; Rossi, D.; Paganetto, G.; Muzzoli, M.; Scalvenzi, L.; Sacchetti, G.; Food Chem. 2009, 114, 1413.

29. Souza, B. de A.; Roubik, D. W.; Barth, O. M.; Heard, T. A.; Enríquez, E.; Carvalho, C. A. L.; Villas-Bôas, J. K.; Marchini, L. C.; Locatelli, J. C.; Persano-Oddo, L.; Almeida-Muradian, L. B.; Bogdanov, S.; Vit, P.; IC 2006, 31, 867.

30. White Júnior, J. W.; Bee World 1994, 75, 104.

31. Evangelista-Rodrigues, A.; Silva, E. M. S.; Beserra, E. M. F.; Rodrigues, M. L.; Cienc. Rural. 2005, 35, 1166.

32. Silva, R. A. da; Rodrigues, L. M. F. M.; Lima, A. de; Camargo, R. da C. $\mathrm{R}$ de; Higiene Alimentar 2006, 20, 90.

33. Welke, J. E.; Reginatto, S.; Ferreira, D.; Vicenzi, R.; Soares, J. M.; Cienc. Rural 2008, 38, 1737. 